\title{
Jet Substructure at HERA
}

\section{Margherita Milite* on behalf of the ZEUS Collaboration}

Address DESY, Notkestrasse 85 22607 Hamburg, Germany

E-mail: milite@mail. desy.dé

ABSTRACT: The internal structure of hadronic jets selected in exclusive and charminduced dijet events in high energy $\gamma p$ interactions and in charged current (CC) deep inelastic $e^{+} p$ scattering (DIS) has been investigated with the ZEUS detector at HERA. The substructure of jets has been studied in terms of the jet shape and the subjet multiplicity. Comparison between the exclusive dijet sample and the charm- (i.e. quark-) induced sample allows the extraction of the jet substructure for gluon jets. Leadinglogarithm parton-shower Monte Carlo calculations provide a good description of the data. The quark-induced jet samples selected from charm-induced dijets and from charged and neutral current (NC) DIS have been compared and found to be similar.

\section{Introduction}

The investigation of the internal structure of jets gives insight into the transition between a parton produced in a hard process and the experimentally observable spray of hadrons. The internal structure of a jet is expected to depend mainly on the type of primary parton, quark or gluon, from which it originated and to a lesser extent on the particular hard scattering process. Moreover, gluon jets are predicted to be broader than quark jets due to the larger colour charge of the gluon. Useful representations of the jet's internal structure are given by the jet shape [i], which represents the fractional energy distribution about the jet axis direction, and the subjet multiplicity [2], representing the number of jet-like objects within a given jet. The measurements presented were performed using data samples collected with the ZEUS detector at HERA during 1995-2000.

\section{Jet substructure in exclusive and charm-induced dijets in $\gamma p$}

Events with two jets of transverse energies $E_{T}^{\text {jet } 1,2} \geq 7,6 \mathrm{GeV}$ and pseudorapidities $-1 \leq$ $\eta^{\text {jet 1,2 }} \leq 2$, reconstructed using the longitudinally invariant $k_{T}$-cluster algorithm in the laboratory frame, were selected. A charm-enriched jet sample was obtained by selecting

\footnotetext{
${ }^{*}$ Speaker.
} 
those events where one of the jets originated from a charm quark, identified by tagging a $D^{*^{ \pm}}$(2010) meson in the final state through the K2 $\pi$ decay mode. The jet with the closest distance in azimuthal angle to the $D^{*}$ meson candidate was associated with the charm meson. The second jet in the event (referred to as the "untagged-charm" jet) represents the unbiased, i.e. not influenced by the selection cuts on the $D^{*}$ meson, jet candidate whose internal properties were investigated. The measurements [3] 3$]$ were performed in the photoproduction regime of $e^{+} p$ interactions. The kinematic region is defined by $Q^{2} \lesssim 1$ $\mathrm{GeV}^{2}$, where $Q^{2}$ is the virtuality of the exchanged photon, and inelasticity $0.2 \leq y \leq$ 0.85. The measured mean subjet multiplicity as a function of the resolution scale $y_{\text {cut }}$ for the exclusive dijet sample is shown in Fig. $i_{-1}^{1}(a)$. The MC predicted $\left\langle n_{s b j}\right\rangle$ is larger for gluon-initiated jets than for quark-initiated jets and the data lie between the two curves, indicating that the dijet sample is a mixture of quark and gluon jets. The measured mean subjet multiplicity for the "untagged-charm" jets is shown Fig. $i_{1}^{1}(\mathrm{~b})$. The Monte Carlo predictions for charm-initiated jets are in very good agreement with the data.

\section{ZEUS}

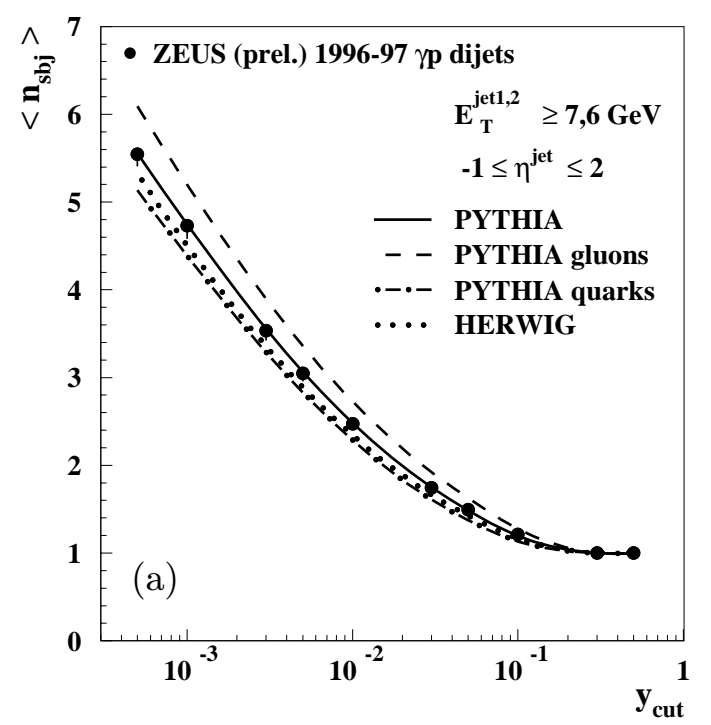

ZEUS

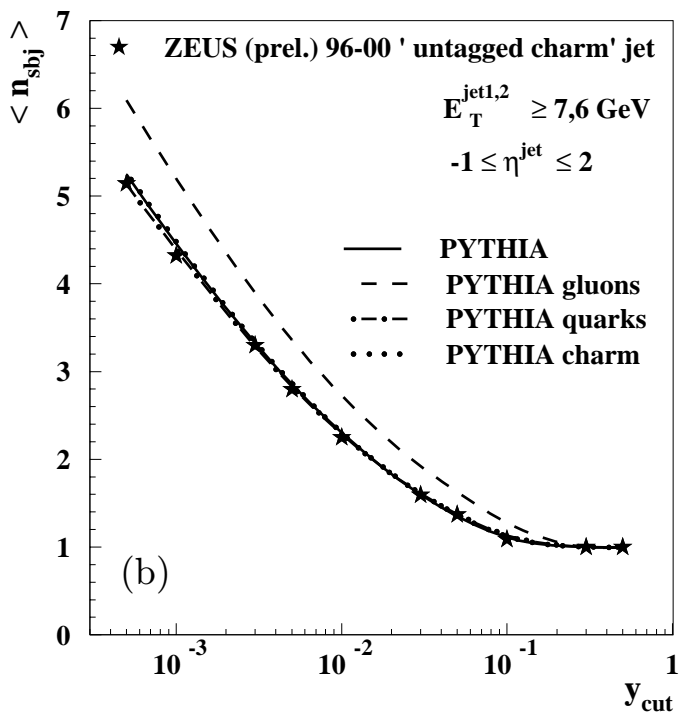

Figure 1: Mean subjet multiplicity $\left\langle n_{s b j}\right\rangle$ as a function of $y_{\text {cut }}$ corrected to hadron level for (a) the exclusive dijet sample and (b) the "untagged charm" jet sample in photoproduction.

Figure $\overline{2}$ shows the differential jet shape as a function of the distance from the jet axis, $r \equiv \sqrt{(\Delta \eta)^{2}+(\Delta \phi)^{2}}$, for the exclusive and the charm-induced jet samples. In this case, the agreement between data and Monte Carlo is also very good. The dependence of the mean subjet multiplicity on $\eta^{\text {jet }}$ for the exclusive and "untagged charm" jet samples and for an inclusive jet sample selected in CC DIS interactions $\left(Q^{2} \geq 200 \mathrm{GeV}^{2}\right)\left[\begin{array}{l}{[1} \\ -1\end{array}\right]$ is shown in Figure $\overline{3} \cdot$. In the exclusive dijet sample, the $\left\langle n_{s b j}\right\rangle$ increases as $\eta^{\text {jet }}$ increases, which is consistent with the predicted increase in the fraction of gluon-initiated jets, $f_{g}$, with $\eta^{\text {jet }}$. In the "untagged charm" jet sample, the results are consistent with the prediction for a pure sample of quark jets for $-1 \leq \eta \leq 1.5$. In the highest $\eta^{\text {jet }}$ bin, the data point shows a deviation from the prediction of a pure sample of quark jets. Since the estimated gluon 
ZEUS

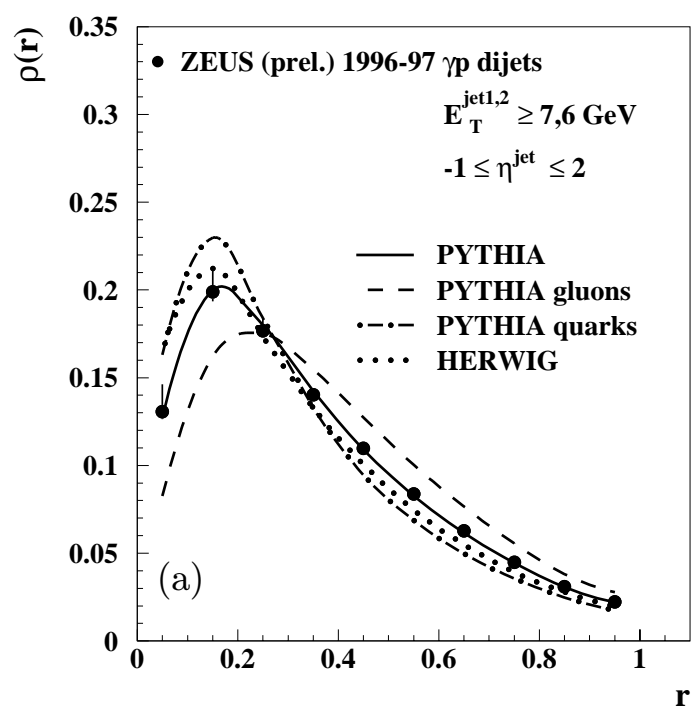

ZEUS

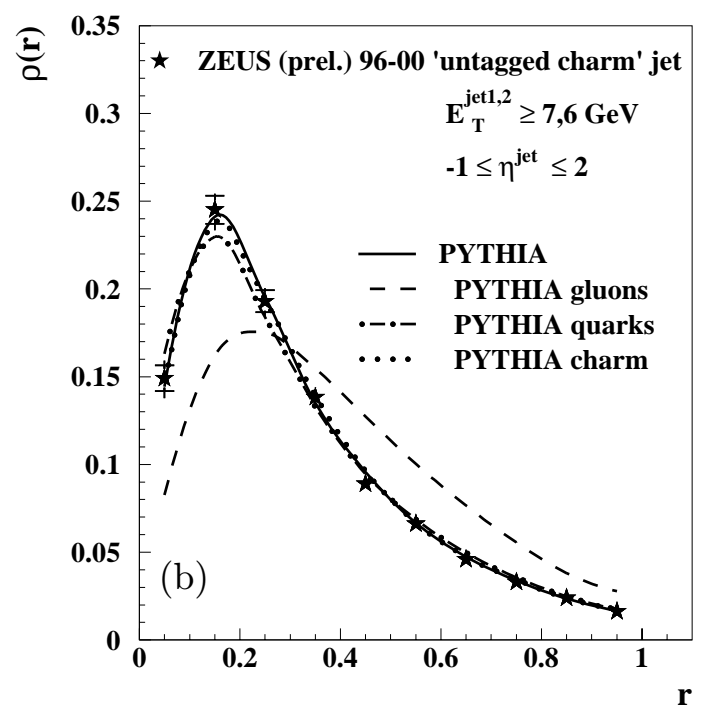

Figure 2: Differential jet shape $\rho(r)$ as a function of $r$ for (a) the exclusive dijet sample and (b) the "untagged charm" jet sample in photoproduction.

contamination to the charm-induced jet sample (mainly due to the "charm excitation" process, $c_{\gamma} g \rightarrow c g$ ) has its largest contribution in the forward region, this deviation could be explained by an increase of $f_{g}$ with $\eta^{\text {jet }}$ in the charm-enriched sample. In the case
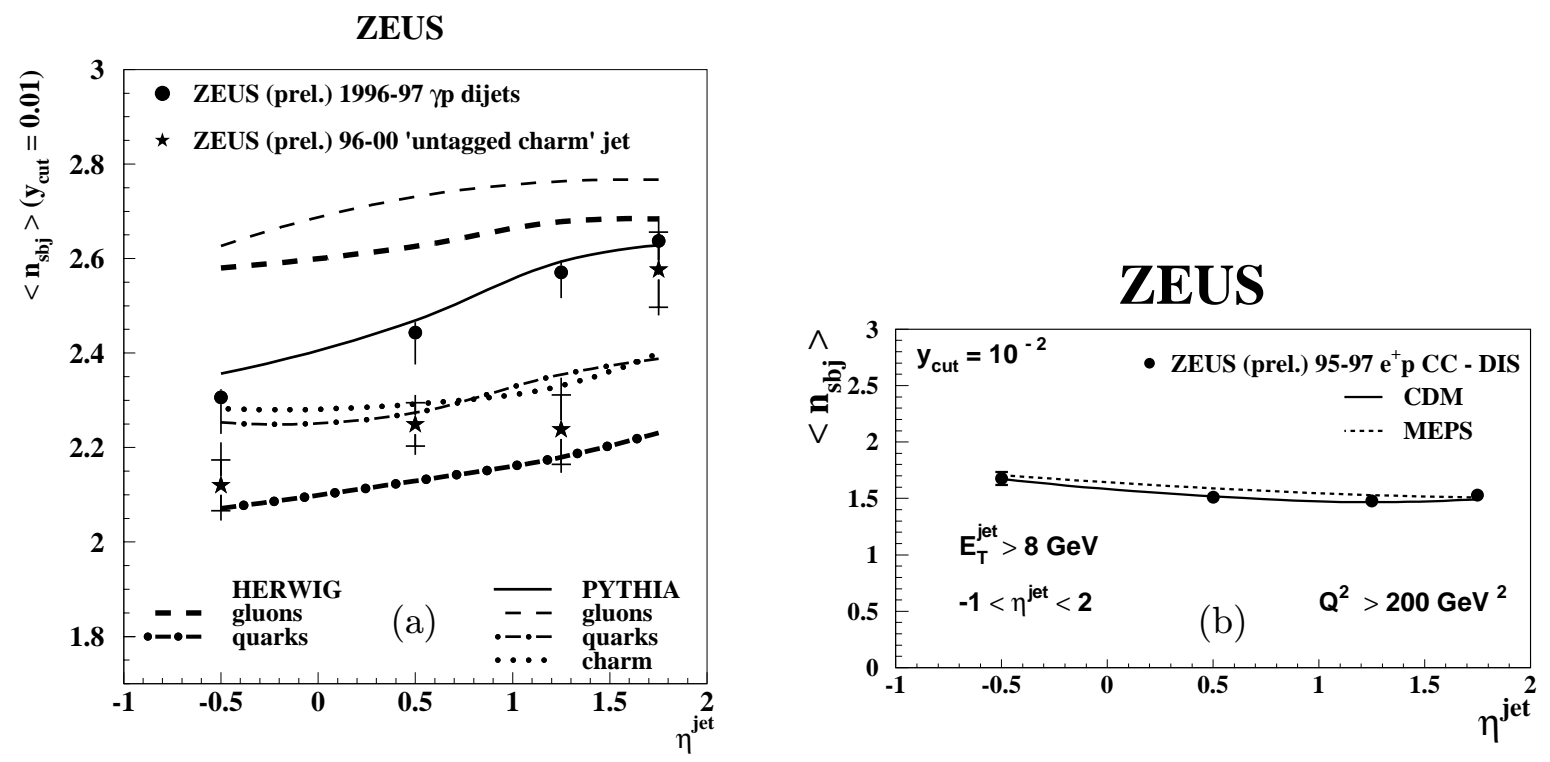

Figure 3: The $\left\langle n_{s b j}\right\rangle$ for a fixed $y_{\text {cut }}=0.01$ as a function of $\eta^{\text {jet }}$ for (a) the exclusive and "untaggedcharm" jet samples in photoproduction and (b) for the inclusive jet sample in CC DIS.

of the inclusive CC jet sample, no $\eta^{\text {jet }}$ dependence is observed (see Figure $\bar{p}_{1}(\mathrm{~b})$ ) which is consistent with the dominance of quark-initiated jets over the entire $\eta^{\text {jet }}$ range. 


\section{Comparison with inclusive jet production in NC and CC DIS}

Figure $\overline{4}(\mathrm{i})$ shows the mean subjet multiplicity as a function of $y_{\text {cut }}$ for the "untagged charm" jet sample compared with an inclusive jet sample in NC DIS $\left(Q^{2} \geq 125 \mathrm{GeV}^{2}\right)$ [i6]" The data points are in very good agreement, especially for values of the resolution scale $y_{\text {cut }} \geq 0.01$ where the charm-mass effects become negligible. Thus, charm-initiated jets are very similar to light quark-initiated jets. The $\left\langle n_{s b j}\right\rangle$ as a function of $E_{T}^{\text {jet }}$ at a fixed $y_{\text {cut }}=0.01$ for inclusive jet samples in NC and CC DIS is presented in Figure this case, the agreement between the data sets is very good. From the comparisons shown, it can therefore be concluded that the evolution of the outgoing partons, which determines the internal structure of the final state hadronic jets, is to a large extent independent of the hard subprocess from which the outgoing partons originate.

\section{ZEUS}

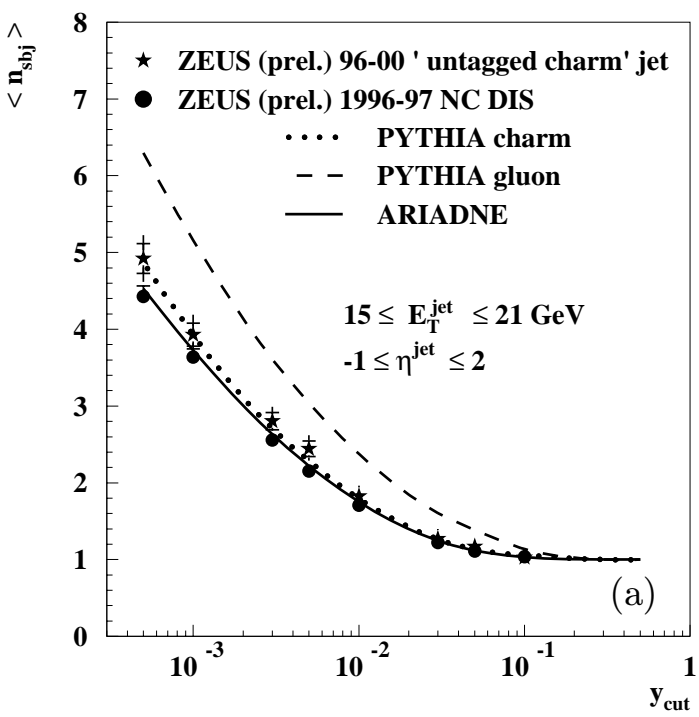

\section{ZEUS}

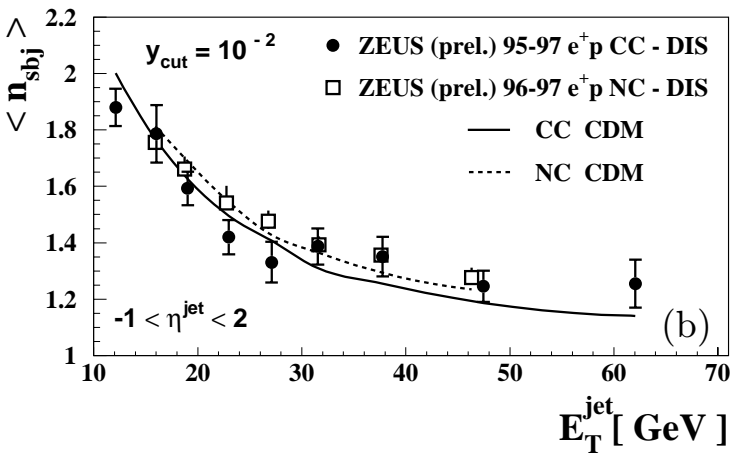

Figure 4: $\left\langle n_{s b j}\right\rangle$ as a function of $y_{\text {cut }}$ corrected to hadron level for (a) the "untagged charm" jet sample in photoproduction compared with the inclusive jet production in neutral current DIS and (b) $\left\langle n_{s b j}\right\rangle$ for a fixed value of $y_{\text {cut }}=0.01$ as a function of $E_{T}^{\text {jet }}$ for inclusive jet production in NC and CC DIS.

\section{Characterisation of gluon-induced jets}

Since the dijet photoproduction sample consists of a mixture of quark and gluon jets, any measured observable $\mathcal{O}$ can be written as: $\mathcal{O}_{\text {dijet }}=f_{q} \cdot \mathcal{O}_{\text {quark }}+f_{g} \cdot \mathcal{O}_{\text {gluon }}$, where $f_{q}$ and $f_{g}=\left(1-f_{q}\right)$ are the fractions of quark and gluon jets in the sample and $\mathcal{O}_{\text {quark }}$ and $\mathcal{O}_{\text {gluon }}$ are the observables for a pure sample of quark and gluon jets, respectively. The charm-induced jet sample at high transverse energy $\left(E_{T}^{\text {jet }} \geq 15 \mathrm{GeV}\right)$ can be considered as a sample of pure quark jets (i.e. $\mathcal{O}_{\text {charm }}=\mathcal{O}_{\text {quark }}$ ). Therefore, the substructure for gluon jets can be extracted by taking the fractions $f_{q}$ and $f_{g}$ from LO Monte Carlo calculations. The fractions $f_{q}$ predicted for a dijet sample with $E_{T}^{\text {jet }} \geq 15 \mathrm{GeV}$ and $-1<\eta^{\text {jet }}<2$ by the 


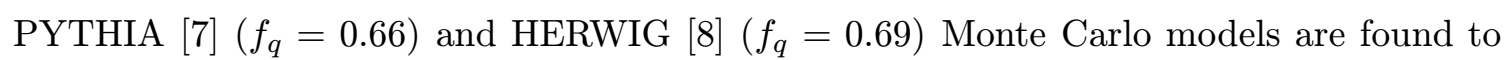
be very similar. The results for the mean subjet multiplicity and the integrated jet shape for gluon jets compared with the measurements for the "untagged-charm" jets are shown in Figure values for the gluon jet substructure. The comparison shows that, as expected, gluon jets are broader than quark jets.

ZEUS

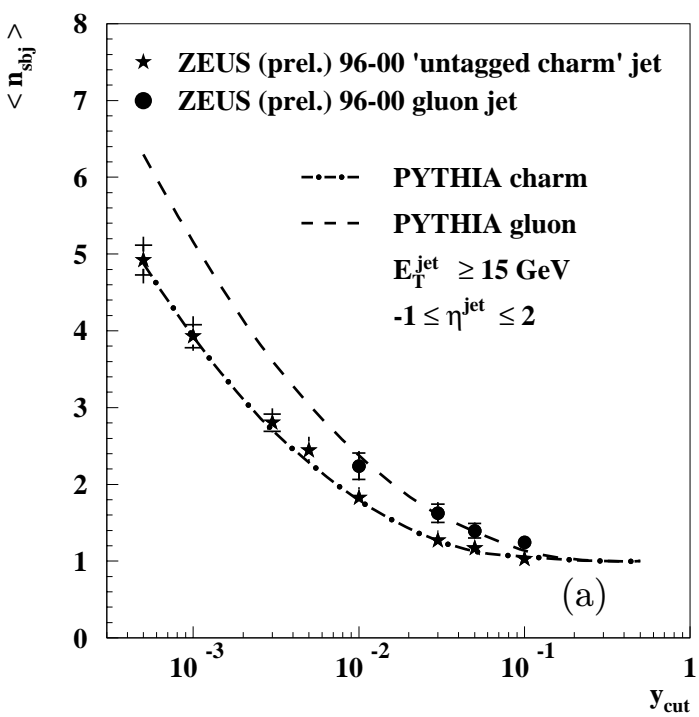

ZEUS

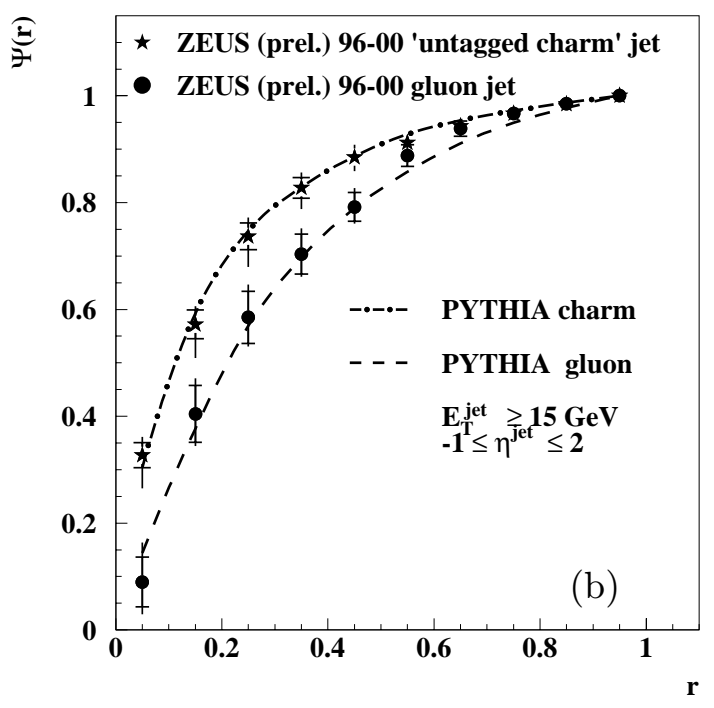

Figure 5: (a) $\left\langle n_{s b j}\right\rangle$ as a function of $y_{\text {cut }}$ and (b) the integrated jet shape as a function of $\mathrm{r}$ corrected to hadron level for gluon and "untagged-charm" jets with $E_{T}^{\text {jet }} \geq 15 \mathrm{GeV}$.

\section{References}

[1] S.D. Ellis, Z. Kunszt and D.E. Soper, Phys. Rev. Lett. 69 (1992) 3615.

[2] S. Catani et al., Nucl. Phys. B377 (1992) 445 and Nucl. Phys. B383 (1992) 419; M.H. Seymour, Nucl. Phys. B421 (1994) 545 and Phys. Lett. B378 (1996) 279.

[3] ZEUS Collaboration, "Measurements of jet substructure in exclusive and charm-induced dijet photoproduction and characterisation of quark and gluon jets" paper 640 submitted to the International Europhysics Conference on High Energy Physics 2001 (EPS-HEP 2001).

[4] ZEUS Collaboration, "Measurements of jet cross sections and jet substructure in charged current deep inelastic scattering at HERA", paper 646 submitted to the EPS-HEP 2001.

[5] S. Catani et al., Nucl. Phys. B406 (1993) 187.

[6] ZEUS Collaboration, "Measurements of jet substructure in neutral current deep inelastic scattering and determination of $\alpha_{S}$ at HERA", paper 641 submitted to the EPS-HEP 2001.

[7] H.U. Bengtsson and T. Sjöstrand, Comp. Phys. Commun. 46 (1987) 43;

T. Sjöstrand, Comp. Phys. Commun. 82 (1994) 74.

[8] G. Marchesini et al., Comp. Phys. Comm. 67 (1992) 465 and hep-ph/9607393. 